\title{
Why should clinical trials be registered?
}

$\Lambda$ friend of ours is considering entering a multicentre phase II placebo-controlled trial of a drug for the treatment of small-cell lung cancer. The patient information sheets, although detailed and clear, contain no promise that the trial findings will ever be published or made available to the public or even to the local trial investigators. Our friend, contemplating his condition and dismal prognosis, is unlikely to ask about this. He knows that the chance of a significant benefit to himself is probably remote (and he may be assigned the placebo), the risks substantial (if in fact he is given the drug) and the inconvenience and fatigue of more clinic visits and tests certain. But, like many patients who enrol in clinical trials, he is motivated by an altruistic hope that he will be contributing to an effort that one day might help patients just like him. And he assumes, as most people would, that regardless of the results of the trial there will be a payoff in scientific understanding.

What our friend may not consider is that the growth of this understanding is impeded by the fact that many data from clinical trials never see the light of day. The results of his trial may not be published for a variety of reasons, such as failure to enrol sufficient patients, funding cuts, parallel discoveries that make the trial irrelevant or, most likely, because the trial results are unfavourable to the sponsor's product. And, should this trial remain unpublished, the public, physicians, editors, systematic reviewers and metaanalysts will be unaware even that it took place - unless it has been entered in an accessible trial registry.

In this issue (see page 1700) ${ }^{1}$ the International Committee of Medical Journal Editors (ICMJE) reiterates and clarifies its previous statement ${ }^{2}$ that registration of clinical trials at their inception will, as of July 1, 2005, become a condition of publication. Failure to register a new trial after that date will oblige CMAJ and other ICMJE member journals along with scores of other journals who have supported the original statement on registration to refuse the subsequent paper for editorial consideration.

Trial registration has a number of benefits. It makes it easier for patients to locate trials for which they might be f eligible and through which they might access new therapies. Registration makes it more difficult to suppress knowledge of investigations begin done, and hence helps to counteract the distorted efficacy and safety profiles that arise from the selective publication of trial results. A registry also helps in the assessment of existing completed trials. Peer reviewers, editors, commentators and individual physicians and patients are able not only to examine published work in a particular field but can also see what is coming down the trial pipeline. For example, editors may be considering a report of a drug trial that shows, against placebo, a clinical benefit but also a substantial but nonsignificant risk of a serious adverse event. A search of the public registry of trials might reveal a similar trial with a sample size sufficient to give more precise estimates of the frequency of adverse events. Clinicians and patients who are aware of the ongoing trial might be advised to wait for its results before using the new drug. The multiple ongoing trials of COX-2 inhibitors are good recent examples.

Similarly, systematic reviewers and meta-analysts who are aware of ongoing trials and their sample sizes can speculate on the possible effects of forthcoming data on their conclusions and analyses. Both government funding agencies and pharmaceutical companies who sponsor trials can use a registry of ongoing trials when making decisions about new trial funding.

The pharmaceutical companies who fund the majority of clinical trials have argued that public disclosure of their ongoing trials in a registry would reveal secret business objectives, infringe intellectual property rights and damage stock value and investor interest. Others argue, however, that by their very nature clinical trials (multicentred, involving multiple investigators and hundreds of patients, and under the continuous scrutiny of Wall Street analysts) are already "public" and that the commercial argument for guarding secrecy is largely specious. Moreover, when all players abide by the same rules of disclosure, none is at a disadvantage.

The current situation, in which clinical trials involving human subjects are conducted behind the veil of "proprietary interest" and are subjected to the scrutiny of scientific review only at the will of their commercial sponsors, is unacceptable on ethical and scientific grounds. There must be a better balance between ensuring the commercial viability of "R \& D" and serving the best interests of patients and the public. The public registration of clinical trials is one way to rebalance the scales. - CMAf

\section{References}

1. De Angelis CD, Drazen JM, Frizelle FA, Haug C, Hoey J, Horton R, et al. Is this clinical trial fully registered? A statement from the International Committee of Medical Journal Editors [editorial]. CMA7 2005;172(13):1700-2.

2. De Angelis C, Drazen JM, Frizelle FA, Haug C, Hoey J, Horton R, et al. Clinical trial registration: a statement from the International Committee of Medical Journal Editors [editorial]. CMA7 2004;171(6):606-7. 